\title{
Dikaiopoiia - Die Lehre von der Rechtfertigung des Sünders im Dialog der Wittenberger Reformation mit dem Patriarchat von Konstantinopel
}

\section{Die Confessio Augustana Graeca und Melanchthons Schreiben an Patriarch Joasaph II. von Konstantinopel vom September}

\section{9}

Vor gut 460 Jahren, im September 1559, schrieb Philipp Melanchthon dem „Allerheiligsten Patriarchen der Gemeinde von Konstantinopel“ einen Brief, ${ }^{1}$ in dem er den Byzantiner darum bittet, nicht den Verleumdungen zu glauben, welche die römischen Feinde der reformatorischen Bewegung andichten, sondern sich einen vorurteilslosen Eindruck von der Wahrheit zu verschaffen, die man unter den Anhängern Luthers bekennt. Ein gewisser Demetrios wird dabei als Zeuge dafür angerufen, dass die Wittenberger Reformation in Theorie und Praxis als orthodox zu gelten habe. Wer war dieser Mann? Ernst Benz hat darauf in seinem Werk „Wittenberg und Byzanz. Zur Begegnung und Auseinandersetzung der Reformation und der östlich-orthodoxen Kirche“"2 eine detaillierte Antwort gegeben. So genügt der Hinweis, dass es sich bei besagtem Demetrios um einen Diakon des Patriarchen von Konstantinopel handelte, der die religiöse und kirchliche Lage in Wittenberg und Deutschland erkunden sollte. Fast ein halbes Jahr weilte er im Hause Melanchthons als dessen persönlicher Gast. Bei seiner Abreise wurde er vom Praeceptor Germaniae mit einer wichtigen Mission an den Patriarchen beauftragt. Zum Zwecke theologischer Verständigung sollte er ihm eine griechische

1 Vgl. CR IX, 922-924.

2 Ernst Benz, Wittenberg und Byzanz. Zur Begegnung und Auseinandersetzung der Reformation und der östlich-orthodoxen Kirche, Marburg 1949, hier bes. 59-93. Vgl. ferner die Angaben bei Georg Kretschmar, Die Confessio Augustana graeca, in: Kirche im Osten. Studien zur osteuropäischen Kirchengeschichte und Kirchenkunde, hg.v. Robert Stupperich, Bd. 20 (1977), 11-39, hier: 11f. Anm. 2.

Ә OpenAccess. (c) 2022 Gunther Wenz, publiziert von De Gruyter. (cc) BY-NC-ND Dieses Werk ist lizenziert unter einer Creative Commons Namensnennung - Nicht kommerziell - Keine Bearbeitung 4.0 International Lizenz. https://doi.org/10.1515/9783110683868-013 
Fassung der Confessio Augustana ${ }^{3}$ als des wichtigsten reformatorischen Bekenntnisses übergeben.

Die Grundlage der Übersetzung der Augustana ins Griechische bildete primär die Editio princeps und zwar zumeist in ihrer lateinischen Version. Allerdings konnte gelegentlich auch die Variata Verwendung finden, wobei in aller Regel die Ausgabe von 1540 bevorzugt wurde. Das ist vor allem im Kontext der Lehre der Fall, auf die im Folgenden die Aufmerksamkeit ausschließlich konzentriert werden soll, weil sie nach reformatorischem Urteil den articulus stantis et cadentis ecclesiae thematisiert: der Rechtfertigungslehre. Die Artikel 4, 5 und 6 der CA Graeca sind ganz oder weitgehend, die Artikel 12 und 20 immerhin zum Teil von der Variata von 1540 her konzipiert. ${ }^{4}$ Das hat sicher auch hermeneutische Gründe; Melanchthon konnte Anlass zu der Meinung haben, die in der Variata entwickelte Rechtfertigungslehre sei für griechisches Denken anschlussfähiger als die einschlägigen Passagen der Editio princeps, welche ganz vom Konstitutionsgeschehen der Rechtfertigung her entwickelt sind, wohingegen die Version von 1540 explizit auf Genese und Realisierung des Rechtfertigungsglaubens reflektiert.

Um durch den Verweis auf Melanchthon und die konstantinopolitanischen Griechen keine Missverständnisse bezüglich der Entstehung der Confessio Augustana Graeca hervorzurufen, muss festgehalten werden, dass ersterer nicht deren Primärautor war und letztere nicht als deren Primäradressaten gelten

3 Eine Kopie des nur noch in wenigen Exemplaren bekannten ursprünglichen Drucks der Confessio Augustana Graeca von 1559, der bei Johannes Oporinus in Basel erfolgt ist, hat mir dankenswerterweise Herr Kollege Prof. Dr. Dr. Dr. h.c. mult. Theodor Nikolaou zur Verfügung gestellt. Ein Nachdruck erschien 1584 in Wittenberg zusammen mit dem Briefwechsel zwischen der Leitung der Württembergischen Kirche und Patriarch Jeremias II. von Konstantinopel in den Acta et Scripta Theologorum Wirtembergensium, et Patriarchae Constantinopolitani D. Hieremiae [...] Graece et Latine ab iisdem theologis edita. Witebergae. In Officina Haeredum Johannis Cratonis, Anno M.D.LXXXIIII (5-53). Der Titel des Erstdrucks nennt als den Urheber der Übersetzung einen gewissen Paulus Dolscius aus Plauen in Sachsen. Schon im 16. Jahrhundert begegnet allerdings auch die später von Ernst Benz, Byzanz, 94-128, erneut vertretene Auffassung, nicht Dolscius, sondern Melanchthon sei der Autor der Übersetzung von 1559. Demgegenüber hat Georg Kretschmar deutlich zu machen versucht, „daß die Grundlage der CA graeca tatsächlich eine von Paulus Dolscius aufgrund der Invariata gefertigte Übersetzung war. Dieser Text ist aber nun, nachdem er bereits gesetzt war, vor der endgültigen Drucklegung noch einmal überarbeitet worden. Hierfür wird man dann allerdings Melanchthon ins Spiel bringen müssen; nur er hatte die Freiheit und die Autorität zu solch tiefen Eingriffen in den Text des Augsburger Bekenntnisses.“ (Kretschmar, Confessio, 21). Zu der lateinischen Version, die Martin Crusius in den Acta et Scripta der CA Graeca beigegeben hat, vgl. Dorothea Wendebourg, Reformation und Orthodoxie. Der ökumenische Briefwechsel zwischen der Leitung der Württembergischen Kirche und Patriarch Jeremias II. von Konstantinopel in den Jahren 1573-1581, Göttingen 1986, 157f. Anm. 15. 4 Vgl. im Einzelnen D. Wendebourg, Orthodoxie, 155-162, hier: 157 Anm. 15. 
können. Der ursprüngliche Übersetzer war vielmehr aller Wahrscheinlichkeit nach $^{5}$ der Tübinger Philologe Paulus Dolscius (Döltsch), der sein Werk in der humanistisch-pädagogischen Absicht, die Kenntnis des Griechischen an einem klassischen Text der Reformation zu befördern, für gebildete Leser seines engeren und weiteren Umkreises konzipierte und dabei jedenfalls den Patriarchen von Konstantinopel in keiner Weise im Sinn hatte. Er und damit die östliche Christenheit kamen als Adressaten erst sekundär in Betracht, wobei der erwähnte Diakon Demetrios als Mediator fungierte und die Bearbeitung der Vorlage des Dolscius durch Melanchthon gesprächsweise nicht unwesentlich mitgestaltet haben dürfte.

Wie immer der Bearbeitungsvorgang im Einzelnen vonstattengegangen sein mag, durch den Adressatenwechsel hatte sich der Sitz im Leben des Textes grundlegend geändert mit der Folge, dass die Dolsciusübersetzung den veränderten Ansprüchen nicht mehr genügen konnte. Die Neugestaltung der rechtfertigungstheologischen Artikel auf der Basis der Variata, die im Abendmahlsartikel bemerkenswerterweise nicht verwendet wird, sowie die Reformulierung einzelner Termini technici der lateinischen Theologensprache dürfte hierdurch motiviert sein, auch wenn sich in letzterer Hinsicht nicht mit Sicherheit nachweisen lässt, welche griechischen Begriffsbildungen schon von Dolscius und welche erst von Melanchthon stammen. Klar ist in jedem Fall, dass der Übersetzungsvorgang in

$5 \mathrm{Zu}$ den Gründen dieser Annahme vgl. im Einzelnen G. Kretschmar, Confessio, 18ff., hier: $20 \mathrm{f}$ : „Die CA graeca schließt sich [...] in ihrem Aufriß offenkundig der Invariata an, etwa dort, wo Melanchthon später die Reihenfolge der Artikel verändert hat. Aber auch die Übersetzung der einzelnen Artikel folgt in der Regel der Invariata, bisweilen selbst dort, wo der Wortlaut der beiden Fassungen eine unterschiedliche theologische Position widerspiegelt wie in Art. 10 (de coena domini). Wäre Melanchthon ohne Einschränkungen als Urheber dieser Übersetzung der Confessio Augustana ins Griechische anzusehen, würde ein derartiges Vorgehen in einem sehr seltsamen Lichte erscheinen. Eine detaillierte Analyse könnte aber nun zeigen, daß der aufgrund der Editio princeps von 1531 übersetzte Text auf weite Strecken hin nach der Variata überarbeitet ist, in der Regel durch Einschübe oder Auslassungen. Art. 4 und 5 (von der Rechtfertigung und vom Predigtamt) haben sogar die Fassung der Variata zur Grundlage. Gerade hier und in den überarbeiteten Partien finden sich dann immer wieder die Paraphrasierungen und Ergänzungen, von denen Benz völlig überzeugend den Eindruck gewonnen hat, daß sie dazu bestimmt seien, Sachverhalte der abendländischen, lateinischen Tradition für griechische Empfänger zu verdeutlichen." Diese und andere Beobachtungen haben G. Kretschmar zu der bereits erwähnten durch D. Wendebourg, Orthodoxie, 156 Anm. 4 bestätigten - Vermutung geführt, Dolscius sei zwar der Urheber der Übersetzung, Melanchthon habe diese aber vor der endgültigen Drucklegung noch einmal gründlich nach Maßgabe seiner theologischen Überzeugungen und unter Berücksichtigung der geplanten konstantinopolitanischen Mission des Demetrios überarbeitet. Kretschmar kann die Confessio Augustana in diesem Sinne eine „Variatissima“ (Kretschmar, Confessio, 22) nennen. 
den Dienst eines um Sachverständigung bemühten hermeneutischen Verfahrens gestellt ist.

Exemplarisch zu verweisen ist in diesem Zusammenhang auf die Umschreibung der Begriffe und Wortgruppen um satisfactio und meritum ${ }^{6}$ sowie auf den reformatorischen Zentralterminus der iustificatio, der einerseits mit den einschlägigen neutestamentlichen Äquivalenten, andererseits mit einem eigens erfundenen Kunstwort wiedergegeben wird: dikaiopoiia. Offenbar glaubte Melanchthon, auf den dieses „auffälligste (Wort) der ganzen CA Graeca“" zurückgehen dürfte, in diesem Fall der bezeichneten Sache nur durch eine direkte Nachbildung des sie genuin bezeichnenden Wortes entsprechen zu können. Auch wenn man zu dem Gesamtresultat gelangen mag, es sei nicht gelungen, eine CA-Version herzustellen, „die Griechen die Lehre des Augsburgischen Bekenntnisses verständlich gemacht hätte“" , so wird man doch den erstmals gemachten Anfang und die Intention Melanchthons zu würdigen haben, den Griechen in theologicis ein Grieche zu sein. Diese Absicht entsprach nicht nur Melanchthons irenischer Natur, sondern auch seinem stark am Erbe der Väter orientierten theologischen Konzept. Sein Schreiben an Patriarch Joasaph II. von Konstantinopel, das er 1559

6 Vgl. Kretschmar, Confessio, $24 \mathrm{ff}$. sowie Wendebourg, Orthodoxie, $159 \mathrm{f}$.

7 Wendebourg, Orthodoxie, 161. Vgl. in diesem Zusammenhang auch die Bemerkung von G. Kretschmar, wonach es Melanchthon spätestens bei seiner Überarbeitung der Dolscius-Übersetzung klar geworden sein muss, „daß ,iustificatio“ ein Begriff speziell der lateinischen Theologie ist, der nicht einfach und selbstverständlich als gemeinchristlich gelten kann“ (Kretschmar, Confessio, 28). Insbesondere fehlte der östlichen Christenheit die abendländische Theorie und Praxis der sakramentalen Buße als Verstehenshorizont der Rechtfertigungslehre, was die Confessio Augustana Graeca nolens volens dazu veranlaßte, die traditionelle rechtfertigungstheologische Thematik spirituell und im Sinne einer Anleitung zum rechten geistlichen Leben zu akzentuieren. Daraus erhellt, „wie sehr die Aufgabe einer Vermittlung der reformatorischen Gewissheiten in Tradition und Sprache der Griechen - wie jede echte Vermittlungsaufgabe - nicht nur Verstehensprobleme im engeren Sinne des Wortes aufwirft, sondern eine neue Klärung der Sache selbst fordert. Es ist für einen abendländischen Theologen leicht, auf dem Hintergrund der ihm vertrauten Verstehenskategorien die Neuakzentuierung der Rechtfertigungslehre in der CA graeca eine Verkürzung zu nennen. Aber es wäre doch auch zu prüfen, ob es nicht ebenfalls eine Verkürzung der Perspektive nach entgegengesetzter Richtung wäre, wenn eine Umsetzung des ,articulus stantis et cadentis ecclesiae' in Spiritualität von vornherein als unangemessen erscheinen sollte. In diesem Zusammenhang wäre dann das Gewicht der Aussagen über das Wirken des Hl. Geistes im Christen, die aus der Variata in die CA graeca übernommen wurden, im Ganzen des griechischen Textes zu prüfen. Und weiter wäre zu fragen, wie sich diese Einführung des Heiligen Geistes zur Trinitätstheologie verhält, bei Melanchthon und im Blick auf die griechischen Adressaten der Übersetzung.“ (Kretschmar, Confessio, 31; zu den rechtfertigungstheologischen Darlegungen der CA Graeca vgl. ferner E. Benz, Byzanz, 118-121).

8 Wendebourg, Orthodoxie, 162. 
zusammen mit der CA Graeca durch Demetrios in den Pharan gebracht haben wollte, ist ein eindrucksvoller Beleg hierfür.

Zentralmotiv des im Jahr vor Melanchthons Tod verfassten Schreibens ist das Bewusstsein der nahen Endzeit. Dieses ist, wie Ernst Benz zurecht konstatiert hat, „die eigentliche Grundlage der ökumenischen Bestrebungen der Reformation und ist dabei viel maßgeblicher als alles angebliche Bedürfnis nach ,Propaganda““9 . In Anbetracht $\mathrm{zu}$ erwartender und bereits manifest gewordener eschatologischer Drangsale gereicht es Melanchthon zum Trost, von Demetrios erfahren zu haben, dass Gott noch immer auf wunderbare Weise eine nicht kleine Gemeinde in Thrakien und Kleinasien und Griechenland erhält, so wie er einst die drei Männer in der chaldäischen Feuerflamme erhalten hatte. Selbiger Demetrios, dem Melanchthon die trostreiche Nachricht über einen Rest Israels im Türkenland verdankt, wird nun zugleich als Zeuge dafür angerufen, dass es in den reformatorischen Gemeinden mit rechten orthodoxen Dingen zugeht.

Demetrios wird, schreibt Melanchthon dem Patriarchen,

berichten können, dass wir die heiligen Schriften, die prophetischen sowohl wie die apostolischen und die dogmatischen Kanones der heiligen Synoden und die Lehre eurer Väter: des Athanasios, des Basileios, des Gregorios, des Epiphanios, des Theodoret, des Eirenaios und derer, die mit ihnen übereinstimmen, in frommer Weise bewahren. Die alten Frechheiten aber des Samosates und der Manichäer und der Mohammedaner und aller Verfluchten, welche die heilige Kirche verwirft, verabscheuen wir ausdrücklich und lehren, daß die Frömmigkeit im wahren Glauben und im Gehorsam bestehe gegen die uns verordneten Gesetze Gottes, nicht aber im Gehorsam gegen den Aberglauben und die selbstgemachten Gottesdienste, welche die ungebildeten Mönche der Lateiner erfunden haben ohne Gottes Gebot. $^{10}$

Dass dem so sei, davon möge sich der Patriarch auf der Basis der Berichte des Demetrios vorurteilsfrei überzeugen lassen. Die dem Schreiben als Anlage beigefügte Confessio Augustana Graeca sollte auf ihre Weise unterstreichen, dass zwischen der Kirche des Ostens und der Reformation grundsätzlicher Konsens auf der Basis des Zeugnisses Alten und Neuen Testaments, der Kanones der altkirchlichen Synoden, der patristischen Tradition und der Absage an die häretischen Bestreiter der Lehrüberlieferungen der Väter bestehe.

9 Benz, Byzanz, 66.

10 CR IX, 923 in der deutschen Wiedergabe von Benz, Byzanz, 66. 


\section{Von Erbsünde und freiem Willen. Der Briefwechsel der Leitung der Württembergischen Kirche mit Patriarch Jeremias II. von Konstantinopel in den Jahren 1573 bis 1581 im Kontext von FC I und II}

Die Wittenberger Sendung von 1559 erreichte ihr Ziel aller Wahrscheinlichkeit nach nicht. Eine Antwort jedenfalls blieb aus. Sie erfolgte erst gut anderthalb Jahrzehnte später, nachdem die Confessio Augustana Graeca ein zweites Mal an den Bosporus geschickt wurde, diesmal nicht von Wittenberg aus, sondern aus der Universitätsstadt Tübingen, einem damaligen geistigen Zentrum des Luthertums. Als Bote fungierte ein junger evangelischer Prediger an der kaiserlichen Gesandtschaft in Konstantinopel namens Stephan Gerlach. Vorausgegangen war ein Briefwechsel zwischen Martin Crusius (1526 - 1607) und Jakob Andreae (15281590) einerseits und Patriarch Jeremia II. (1536-1595) andererseits. Bei Martin Crusius (Kraus) handelt es sich um einen nahe Bamberg geborenen, seit 1559 als Sprachlehrer und Homerkommentator in Tübingen wirkenden Gräzisten. Jakob Andreae war kein Geringerer als der Hauptverfasser der Konkordienformel und der wichtigste Redaktor des Konkordienwerkes von 1580, mit dem die Einigung des zerstrittenen Luthertums bewirkt werden sollte und jedenfalls z.T. auch tatsächlich bewirkt wurde. Der 1572 zum Patriarchen von Konstantinopel gewählte Jeremias hatte sein Amt bis zu seinem Tode inne, allerdings unterbrochen durch zweimalige Absetzung bzw. politische Verbannung. Auf die slawischen orthodoxen Kirchen übte er großen Einfluss aus; mit seiner Zustimmung wurde 1589 das Moskauer Patriarchat errichtet.

Nachdem Jeremias II. sie mit Schreiben vom 16. September 1574 empfangen hatte, entsprach er der von Crusius und Andreae geäußerten Bitte, die Confessio Augustana Graeca wohlwollend auf ihre Rechtgläubigkeit hin zu überprüfen. Im vierten Jahr seines Patriarchats richtete er am 15. Mai 1576 ein erstes theologisches Schreiben an die Tübinger Professoren, indem er ausführlich Konsens und Dissens mit den evangelischen Glaubensartikeln erläuterte, um schließlich die sieben ökumenischen Konzile und die von der Gesamtgemeinde rezipierte Schriftinterpretation der Väter als die wahrheitsverbürgende Autorität seiner Lehre zu benennen. Der Dissens betrifft vornehmlich folgende Themen: 1. das filioque, das von Jeremias nicht nur formal als unstatthafter Zusatz zu einem sakrosankten Konzilstext, sondern auch inhaltlich als trinitätstheologisch unangemessen abgelehnt wird; 2. die Willensproblematik, in Bezug auf die der Patriarch die Be- 
fürchtung äußert, die reformatorische Lehre von der Rechtfertigung sola gratia, sola fide und per solum Christum verkenne, dass das Heil nicht willenlos, sondern willentlich, nicht ohne tätige Mitwirkung, sondern auf synergistische Weise empfangen werde; 3. die Heiligenfrage, die auf die Frage der Fürbitte der Heiligen, ihrer Anrufung um Fürbitte sowie ihrer möglichen Mittlerfunktion fokussiert wird; 4. die Bilderverehrung; 5. das Mönchtum; 6. die Auseinandersetzung um Begriff und Zahl der Sakramente und schließlich 7. das Verhältnis von Schrift und Tradition. Vor allem diese Kontroversthemen wurden in den folgenden Briefwechsel verhandelt.

Am 18. Juni 1577 replizierten die Tübinger ${ }^{11}$ auf das Schreiben des Patriarchen vom Mai des vorangegangenen Jahres. Es folgte im Mai 1579 ein weiteres Lehrschreiben des Patriarchen, das am Johannistag 1580 von Tübingen aus beantwortet wurde. Mit seinem dritten Brief vom 6. Juni 1581 setzte Jeremias der theologischen Korrespondenz ein Ende:

Wir bitten Euch, uns weiter keine Mühe mehr zu machen und nichts mehr über diese selben Dinge zu schreiben und zu schicken. Da ihr ja die Leuchten und Lehrer der Kirche bald so, bald anders behandelt. Ihr ehrt und haltet sie hoch mit Worten, mit Taten aber verwerft ihr sie. Unsere Waffen bezeichnet ihr als unbrauchbar; dabei sind es ihre heiligen, göttlichen Worte, mit denen auch wir euch zu schreiben und zu widersprechen vermochten. So habt für euren Teil uns der Sorgen entbunden. Geht nun euren Weg! Schreibt uns nicht mehr über Dogmen, sondern allein um der Freundschaft willen (philias de mones heneka; amicitiae tantum causa), wenn ihr das wollt. Lebt wohl! ${ }^{12}$

11 Unterzeichnet haben Martin Crusius und - in Vertretung des abwesenden Andreae - Lucas Osiander, der Sohn von Andreas Osiander. Zu den Unterzeichnern des zweiten (und dritten) Tübinger Antwortschreibens, zu denen neben den Genannten u. a. Jacob Heerbrand zählte, vgl. E. Benz, Byzanz, $95 \mathrm{f}$.

12 Acta et Scripta, 370. Die Tübinger brachten 1584 den gesamten Briefwechsel einschließlich ihrer Antwort auf das dritte Sendschreiben des Patriarchen in den Anm. 2 erwähnten Acta et Scripta an die Öffentlichkeit. (Zu den sonstigen Inhalten vgl. E. Benz, Byzanz, 94-96.) Zu dessen im selben Jahr in Basel erschienener Darstellung in der Turcograecia von Martin Crusius vgl. D. Wendebourg, Orthodoxie, 359-370. In deutscher Übersetzung ist der Briefwechsel in weiten Teilen wiedergegeben in: Wort und Mysterium. Der Briefwechsel über Glauben und Kirche 1573 bis 1581 zwischen den Tübinger Theologen und dem Patriarchen von Konstantinopel, hg.v. Außenamt der EKD, Witten 1958. Dort (11-27) finden sich auch knappe einführende Hinweise zu den Etappen des evangelisch-orthodoxen Gesprächs im 16. Jahrhundert im Zusammenhang der Weltereignisse, zur ökumenischen Bedeutung des Briefwechsels im Zusammenhang mit den hochbyzantinischen orthodoxen Denkmälern des 14. und 15. Jahrhunderts sowie zu seiner Publikation und wissenschaftlichen Bearbeitung. Eine englische Übersetzung bietet G. Mastrantonis, Augsburg and Constantinople. The Correspondence between the Tübingen Theologians and Patriarch Jeremiah II of Constantinople on the Augsburg Confession, Brookline/Mass. 1983. Zum Hintergrund und geschichtlichen Verlauf des Briefwechsels vgl. D. Wendebourg, Orthodoxie, 18- 
Bevor am rechtfertigungstheologischen Themenkreis der inhaltliche Verlauf der Korrespondenz exemplifiziert werden soll, ${ }^{13}$ sei zunächst stichwortartig die Fluchtlinie markiert, auf die hin die Argumentationslinien der Tübinger konvergieren. Dies hat am Beispiel der Konkordienformel zu geschehen, als deren Vater Andreae gilt und durch deren im Mai/Juni 1577 - also exakt zum Zeitpunkt der ersten Replik vom Neckar an den Bosporus - erfolgten Abschluss die theologische Position auch der übrigen Tübinger bestimmt ist. Die Entstehungsgeschichte der Formula Concordiae ${ }^{14}$ gehört in den Zusammenhang der Konfessionalisierung der westlichen Christenheit in der zweiten Hälfte des 16. Jahrhunderts, in der einerseits der tridentinische Katholizismus Gestalt annahm, andererseits die Reformationskirchen $\mathrm{zu}$ jenen konfessionellen Formationen sich ausdifferenzierten, die seither die denominationelle Landschaft bestimmen. Das vorkonkordistische Luthertum wurde nach dem Tod des Reformators bekanntlich durch eine Reihe von Streitigkeiten erschüttert, deren weitgehende Beilegung die wesentliche Leistung des Konkordienwerkes von 1577/80 ist.

Zwei Lager bildeten sich seit den schwierigen Interimszeiten der späten 1540er Jahren innerhalb der Wittenberger Reformation aus: auf der einen Seite standen die sog. Gnesiolutheraner, die von ihren Gegnern als Flacianer apostrophiert wurden, auf der anderen die Philippisten, die sich im Wesentlichen aus Melanchthonschülern rekrutierten. Zwar waren die Lager von Anfang an nicht einheitlich, so dass sich künftige Friktionen ahnen ließen. Gleichwohl sind im Blick auf die seit Interimszeiten anhebenden Auseinandersetzungen Frontverläufe durchaus erkennbar: neben Auseinandersetzungen um Abendmahlslehre, Christologie und Prädestination handelt es sich dabei um die sog. majoristischen, antinomistischen, synergistischen und osiandrischen Streitigkeiten. Weil beide für den rechtfertigungstheologischen Diskurs zwischen Tübingen und Konstantinopel von besonderer Bedeutung sind, seien hier die majoristischen und syn-

151. Bei den nach Stil und Methode je nach Absender sehr unterschiedlich gestalteten Schreiben handelt es sich von Seiten des Patriarchen im Wesentlichen um Zitatkompilationen, von Seiten der Tübinger um mehr oder minder systematische Abhandlungen (vgl. Wendebourg, Orthodoxie, 151 ff.). Zum autoritativen Status der Lehrschreiben des Patriarchen innerhalb der Orthodoxie vgl. D. Wendebourg, Orthodoxie, $398 \mathrm{ff}$.

$13 \mathrm{Zu}$ den sonstigen Streitfragen vgl. D. Wendebourg, Orthodoxie, 207-346. Sie betreffen, wie erwähnt, vor allem den Ausgang des Heiligen Geistes (a.a.0., 212-223), das Verhältnis zu den Heiligen und den Umgang mit kirchlichen Bildern (a.a.O., 253-265), das Mönchtum (a.a.O., 266272) sowie das weite Feld der Theorie und Praxis der Sakramente (a.a.O., 272-334) und der Traditionsproblematik (a.a.O., 334-346).

14 Vgl. Gunther Wenz, Theologie der Bekenntnisschriften der evangelisch-lutherischen Kirche. Eine historische und systematische Einführung in das Konkordienbuch, Bd. 2, Berlin/New York 1998, 467-539. 
ergistischen Auseinandersetzungen und ihre Lösung in der Konkordienformel paradigmatisch in Betracht gezogen.

Ist der majoristische wie übrigens auch der antinomistische Streit primär am Realisierungszusammenhang der Rechtfertigung orientiert, so betrifft die Synergismuskontroverse vor allem die Konstitutionsbedingungen. Vorauszuschicken ist, dass Melanchthon seine ursprüngliche Lehre strenger göttlicher Alleinwirksamkeit im Laufe der Zeit dahingehend abgewandelt hatte, dass er von drei bei der Bekehrung des Menschen zusammenwirkenden causae sprach, nämlich vom Wort, vom Heiligen Geist und von dem die eigene Schwachheit tätig bekämpfenden Willen. Als der Melanchthon-Schüler Johannes Pfeffinger im Jahre 1555 diese Auffassung, deren wesentlicher Gehalt im Leipziger Interim Aufnahme gefunden hatte, nicht nur verteidigte, sondern in der Absicht, ein ursächliches Mitwirken des Menschen bei der Entscheidung seines Heils bzw. seines Unheils zu gewährleisten, weiter verschärfte, trat ihm neben Amsdorff, Schnepff und Flacius anfangs auch Viktorin Strigel entgegen. Strigel war einer der ersten Professoren an der Universität Jena, die 1548 im ernestinischen Sachsen als Ersatz für die infolge der Schmalkaldischen Niederlage mit Kurwürde und Kurkreis an die Albertiner verloren gegangene Universität Wittenberg gegründet worden war. Als 1556/57 auch Flacius eine Professur in Jena erhielt, kam es zwischen beiden bald zu heftigen Kontroversen, zumal Strigel sich dem von Flacius und seinen Anhängern erarbeiteten und zur gesetzlichen Lehrnorm im Herzogtum Sachsen erhobenen Weimarer Konfutationsbuch von 1559 widersetzte und dafür mehrere Monate inhaftiert wurde.

Auch die vom Herzog im August 1560 anberaumte Weimarer Disputation führte zu keinem Ausgleich, wohl aber zum beginnenden Niedergang des theologischen Sterns von Flacius. Während Strigel die These vertrat, die geschöpfliche Substanz des Menschen als eines vernunft- und willensbegabten Lebewesens sei durch die Erbsünde gleich einem mit Zwiebelsaft bestrichenen Magneten geschwächt, aber nicht gänzlich verdorben, steigerte Flacius seine Auffassung von der gänzlichen Verderbnis des postlapsarischen Menschen zu der Annahme, durch Adams Fall sei die Erbsünde die Substanz der menschlichen Natur geworden. Zwar unterschied er dabei zwischen substantia materialis als möglicher Bezugsgröße des Guten und der forma substantialis als Trägerin des Bösen und bestritt überdies der Erbsünde eine eigene Subsistenz. Doch konnten ihn solche Differenzierungen nicht mehr vom Verdacht heterodoxer Übertreibung der Orthodoxie befreien. Seither wurde er - und zwar nicht nur unter den Philippisten, sondern auch von wesentlichen Teilen des gnesiolutherischen Lagers - als Ketzer betrachtet.

Die Konkordienformel versuchte einen Mittelweg zwischen Strigel und Flacius einzuschlagen und die Einseitigkeiten beider zu vermeiden, wobei trotz antifla- 
cianischer Verdikte durchaus die gnesiolutherische Perspektive dominiert. In diesem Sinne grenzt sich FC I (,Von der Erbsünde“) in schroff antipelagianischer Absicht gegenüber allen Positionen ab, die dem postlapsarischen Menschen Restbestände eines soteriologischen Eigenvermögens zudenken. Das peccatum originale verderbe nicht nur einen Teil des Menschen, sondern dessen ganze Natur und bewirke manifeste Feindschaft wider Gott. Wenn gleichwohl gegen mögliche manichäische Implikationen des Flacianismus gesagt wird, die Erbsünde vertilge nicht die menschliche Wesensnatur, dann geschieht dies ausschließlich in der Absicht, den Menschen auf seine kreatürliche Bestimmung zu verpflichten und die Zurechnung seiner Sünde als Schuld zu gewährleisten. Um die Fatalisierung der Sünde zu einem gleichsam naturhaften Geschick zu verhindern, muss auch unter postlapsarischen Bedingungen zwischen kreatürlichem Wesen des Menschen und der alles andere als unwesentlichen Verkehrtheit seiner Sünde unterschieden werden. Ob diese Unterscheidung mittels der Differenzierung von substantia und accidens angemessen geleistet werden kann, ist nach Urteil der Väter der Konkordienformel primär eine terminologische, den modus loquendi betreffende, sachlich hingegen zweitrangige Frage. Sachlich entscheidend hingegen ist der Hinweis in Ep I,10, demzufolge zwischen der kreatürlichen Wesensnatur des Menschen und dem Unwesen der Erbsünde, welcher er schuldhaft verfallen ist, niemand heilsam scheiden kann als Gott allein, dessen in der Kraft des Heiligen Geistes manifeste Offenbarung in Jesus Christus die Voraussetzung für beider erkenntliche Unterscheidung ist. Damit ist erneut klargestellt, dass dem postlapsarischen Menschen sowohl ontologisch als auch gnoseologisch jedes Vermögen fehlt, sein Heil selbsttätig zu besorgen.

Dieser Sachverhalt wird durch die Willenslehre in FC II „Vom freien Willen oder menschlichen Kräften“ voll bestätigt. Sie ist auf das kontroverse Thema konzentriert, was Verstand und Wille des gefallenen und unwiedergeborenen Menschen in dessen conversio und regeneratio zu leisten vermögen, wenn Gottes Wort gepredigt und die Gnade Gottes angeboten wird. Vorausgesetzt ist dabei, dass der postlapsarische Mensch zwar ein gewisses, in seiner Indifferenz sittlich uneindeutiges Willensvermögen besitzt, um sich von Tieren unterscheiden und in einer Menschenwelt bewegen zu können, dass er aber schlechterdings unfähig ist, durch Verstandes- und Willenstätigkeit Gottes Gnade zu erwirken. Kann er in sie zumindest durch eigenes Vermögen einwilligen und ihrer Zusage von sich aus zustimmen? Die Antwort von FC II auf diese Frage fällt differenziert aus: Zwar zwingt Gottes Gnade nicht auf naturkausale Weise und wirkt auf den Menschen daher nicht wie ein Keil auf den Klotz. Der Empfang der Gnade vollzieht sich durchaus im Modus der Freiheit und destruiert nicht das Aufnahmevermögen des Menschen, sondern erfüllt es. Eine menschliche capacitas passiva bezüglich der Gnade ist FC II daher durchaus zu attestieren bereit; aber dabei handelt es sich 
recht eigentlich nicht um eine Fassungskraft des Menschen, weil das Gnadengeschehen tanquam in subjecto patiente ${ }^{15}$ wirksam ist. Der die Gnade im reinen Empfangen des Glaubens hinnehmende Mensch verhält sich zu ihr pure passive, nämlich so, dass er sie sich dankbar gefallen lässt. Jeder Reflex auf ein dem Gnadengeschehen vorgegebenes soteriologisches Eigenvermögen ist damit obsolet. Der Glaube weiß, dass Gott beides wirkt: velle et perficere, Wollen und Vollbringen. Unter diesem Aspekt kann der postlapsarische Mensch samt seinem Willen und Verstand nicht anders in Betracht kommen denn als subjectum convertendum, als ein „Subjekt“, das durch das Evangelium aus seiner Verkehrtheit bekehrt und aus seiner Unfreiheit zur Freiheit der Kinder Gottes zu befreien ist. Damit ist die Perspektive benannt, in welcher die Tübinger mit dem konstantinopolitanischen Patriarchen Jeremias brieflich über das Rechtfertigungsereignis und seine Prämissen diskutieren.

Während Jeremias II. in seinem ausführlichen Kommentar zu den einzelnen Artikeln der Confessio Augustana Graeca vom 15. Mai 1576 CA 2 und 19 hamartiologisch ohne Vorbehalte akzeptierte, ließ er gleichwohl bereits in sündentheologischer Hinsicht keinen Zweifel daran aufkommen, dass der Mensch vermöge seines Willens die Sünde grundsätzlich meiden und das geforderte Gute mit Gottes Hilfe selbsttätig tun könne. Andernfalls werde die Sünde, so der Patriarch zu CA 19, fatalisiert, und zu einem Naturdatum erklärt, das Schicksal sei, ohne als Schuld zugerechnet werden zu können. Nicht weil er von Natur aus nicht anders könne, sondern weil er das Gute nicht wolle und das dem Guten widerstrebende Böse wähle, sündige der Sünder. Analoges ergibt sich in soteriologischer Hinsicht: Gott zwingt den Menschen nicht zum Heil, sondern er beruft Willige in sein Reich. Die Unwilligen aber sind, so sie Gott sich selbst überlässt, an ihrer Heillosigkeit selbst schuld, weil sie diese durch ihre Unwilligkeit Gott gegenüber bewirkt haben. Die Ausführungen über den freien Willen im Zusammenhang des Kommentars zu CA 18 bekräftigen diese Auffassung. Obwohl Gnade, so wird unter Berufung auf den heiligen Chrysostomos gesagt, rettet die Gnade Willige und nicht nach Weise einer gratia irresistibilis. Zwar stehe alles bei Gott, doch nicht so, dass darüber der freie Wille des Menschen Schaden leide. „Bei uns also steht es und bei Ihm! Wir müssen zuerst das Gute wählen, und dann bringt Gott das Seine hinzu. Er kommt unsern Entscheidungen nicht zuvor, damit unser freier Wille nicht verletzt wird. Wenn wir aber unsere Wahl getroffen haben, dann bringt Gott uns seine große Hilfe."16 Wenn Paulus Phil 2,13 sage, dass Gott Wollen und Vollbringen wirke, meine er nicht, dass Gott das Wollen recht eigentlich schaffe,

15 Vgl. BSLK, 910, 16.

16 Acta et Scripta, 114. 
sondern helfend unterstütze und befördere. Der Satz, dass Gott in uns das Wollen wirke, stelle also den freien Willen des Menschen nicht in Abrede, sondern setze ihn voraus.

Dies gefiel den Tübingern nicht: zwar lehnen auch sie jede Form der Fatalisierung der Sünde und der Naturalisierung des Heilsgeschehens ab. Dennoch bekräftigen sie die Annahme, dass der postlapsarische Mensch, also der Mensch wie er sich faktisch vorfindet, ausnahmslos nicht über den Willen verfügt, das göttliche Gut zu wählen, sondern der Güte Gottes und damit zugleich der kreatürlichen Bestimmung seiner selbst und seiner Welt willentlich widerstrebt. Als konstitutives Datum des Heilsgeschehens scheidet das liberum arbitrium somit aus, ja es ist im Gegenteil so, dass das soteriologische Insistieren auf der Wahlfreiheit des Menschen als ein Unheilsdatum qualifiziert wird. Was der Apostel zu den Philippern sagt, will nach Urteil der Tübinger durchaus stricte dictu verstanden sein: „Nicht nur das Gute vollbringen, sondern auch das Gute wollen, ist Gottes Wirkung in uns. "17 Es ist mithin nicht so, dass die Menschen, denen Gottes Gnade in Jesus Christus begegnet, zuerst das Gute wählen, und dann Gott ihnen das Seine hinzufügt. Kraft seines Geistes wirkt Gott vielmehr alles in allem, wenngleich nicht auf naturhafte Weise, sondern im Modus göttlicher Freiheit, welche den Menschen aus der Unfreiheit und Sklaverei seiner Sünde zu sich und seiner Bestimmung befreit. Diese Befreiung von sich aus zu leisten, ist der Mensch auch nicht ansatzweise in der Lage. Der Ansatz bei der unmittelbaren Selbstbestimmung des Menschen in Form eines selbstverständlich vorausgesetzten liberum arbitrium indifferenter Wahlfreiheit ist vielmehr bereits in sich ein Indiz für den abgründigen Fall des Menschen. Denn auf einem indifferenten Wahlvermögen dem Guten gegenüber zu insistieren, ist bereits Wille zum Bösen. Von daher muss, um es zu wiederholen, das liberum arbitrium aus dem Begründungszusammenhang der Soteriologie ausgeschieden werden. Das betreiben die Tübinger konsequent mit der Folge, dass ihnen der Sünder trotz physischen Lebens als ein geistlich Toter gilt. Wie ein toter Körper nichts will und nichts tut, sondern nur üblen Geruch verbreitet, so kann der geistlich erstorbene Mensch, wenn er nicht von Gott auferweckt und wiedergeboren wird, nichts Gutes wählen und nichts Gutes tun. Der status controversiae zwischen Konstantinopel und Tübingen in der Sünden- und Willensfrage ist damit bestimmt. Er wird in der weiteren Korrespondenz nur mehr bestätigt, ohne dass wirklich neue Argumente erkennbar würden.

17 Acta et Scripta, 164. 


\section{Von Glaubensgerechtigkeit und guten Werken. Der Briefwechsel der Württemberger mit Jeremias II. im Kontext von FC III und IV}

Kann von einem verbleibenden soteriologischen Eigenvermögen des sündig in sich verkehrten Menschen und von einer soteriologischen Basisfunktion eines menschlichen liberum arbitrium nach Urteil der Tübinger in keiner Weise die Rede sein, so verbleibt in zweiter Hinsicht zu bedenken, was der Wille der Wiedergeborenen in geistlichen Dingen vermag. Diese Frage betrifft, wenn man so will, den Realisierungszusammenhang der Rechtfertigungslehre und dabei insbesondere das Problem der guten Werke. Binnenlutherisch wurde dieser Themenkreis u.a. im sog. majoristischen Streit kontrovers verhandelt. Der majoristische Streit verdankt seinen Namen einem seiner Hauptprotagonisten, dem Melanchthonschüler und entschiedenen Philippisten Georg Major, seit 1544 Theologieprofessor in Wittenberg. Wie sein Lehrer intensiv an der Autorität der altkirchlichen Väter und an der humanistischen Forderung sittlichen Christentums orientiert lehrte er die Notwendigkeit guter Werke zur Seligkeit. Damit wollte er keinem meritorischen Rechtfertigungsverständnis Vorschub leisten, sondern lediglich betonen, dass die Früchte des Glaubens für dessen Bewahrung unerlässlich seien. Gleichwohl bezichtigten ihn die Gnesiolutheraner der Preisgabe der ursprünglichen Einsicht der Reformation. Um jeden noch so subtilen Pelagianismus gänzlich auszumerzen, verstieg sich Nikolaus Amsdorff gar zu der - zum Titel eines Traktats (1559) erhobenen - Aussage, „dass diese Propositio ,gute Werke sind zur Seligkeit schädlich' eine rechte, wahre, christliche Propositio sei, durch die Heiligen Paulum und Lutherum gelehrt und gepredigt“. Nicht weniger deutlich, doch differenzierter äußerte sich Flacius.

Abermals ist die Konkordienformel um Vermittlung bemüht, wobei erneut die gnesiolutherische Tendenz gegenüber dem Philippismus dominiert. Die dezidiert antisynergistische Perspektive bezüglich der Prämissen des Rechtfertigungsgeschehens wird in Bezug auf dessen Folgezusammenhang beibehalten. Trahit Deus, sed volentem trahit ${ }^{18}$. Dieser Satz wird in FC II zwar als nicht falsch, aber als in hohem Maße erläuterungsbedürftig bezeichnet. Nicht anders stellt sich die Angelegenheit im Kontext der Lehre von der Gerechtigkeit des Glaubens und von den guten Werken dar, wie FC III und IV sie entwickeln. Zwar wirkt der bekehrte Wille am Heiligungswerk Gottes mit, aber die Mitwirkung hängt ausschließlich an der wirksamen Wirklichkeit des Heiligen Geistes, in welchem sie die Bedingung

18 BSLK, 908,2. 
ihrer Möglichkeit, ihren alleinigen Konstitutions- und Erhaltungsgrund findet. Weder Anfang noch Vollzug der Bekehrung sind daher in das Vermögen des Menschen gestellt. Das sola gratia steht nicht nur hinsichtlich der dem Rechtfertigungsgeschehen vorhergehenden, sondern auch hinsichtlich der ihm nachfolgenden Werke in Geltung. Dessen ist der Rechtfertigungsglaube gewärtig und gewiss; er wird deshalb auch sich selbst nicht als Werk wissen, sondern jenseits aller Selbstsicherheit ganz aus dem Vertrauen auf Gott leben, auf welches sich zu verlassen sein Wesen ausmacht. Es gilt der rechtfertigungstheologische Heilsgrundsatz: absque ullo respectu praecedentium, praesentium aut consequentium nostrorum operum ${ }^{19}$.

Was die nachfolgenden Glaubenswerke betrifft, so haben sie nach FC IV das sola gratia des Rechtfertigungsartikels nicht nur zur anfänglichen, sondern zur durchgängigen Voraussetzung. Hingegen dürfen die guten Werke, sosehr sie gottgeboten und insofern nötig sind, weder zum Konstitutions- noch zum Erhaltungsgrund des Glaubens erklärt werden. Weisen wohl schuldig gebliebene Werke auf schwindenden oder gar fehlenden Glauben hin, so können sie umgekehrt niemals die Gewissheit des Glaubens begründen. In der Konsequenz dessen gelangt die Konkordienformel zu der These, dass der Glaube ohne Werke rechtfertige. Damit ist zwar nicht deren Überflüssigkeit behauptet, aber entschieden in Abrede gestellt, dass Werke das Rechtfertigungsgeschehen begründen oder auch nur mitbegründen können. Wo Werke in dieser Absicht erbracht werden, sind sie nicht nur unnütz, sondern schädlich. Nützlich und förderlich und damit ihrem Begriff als gute Werke entsprechend sind sie hingegen nur, wenn sie die im Rechtfertigungsglauben mit Gott versöhnte Person in Dankbarkeit gegenüber der göttlichen Gnade und in der Freiwilligkeit dankbarer Spontaneität vollbringen. Erst wenn die Person des Menschen in der Exzentrizität des Glaubens ihres verlässlichen Gründens in Gott innewird und durch das Rechtfertigungsevangelium Jesu Christi in der Kraft des göttlichen Geistes von der Verkehrtheit der Sünde freikommt, um zu sich selbst und zu seiner gottgegebenen Bestimmung zu gelangen, kurzum: nur wenn die Person des Menschen vor Gott gerechtfertigt ist, sind auch menschliche Werke richtig. Aus diesem Kontext heraus will schließlich auch die Damnation verstanden sein, derzufolge die Lehre zu verwerfen sei, „daß gute Werk nötig sein zur Seligkeit. Item, daß niemand jemals ohne gute Werk sei selig worden. Item, daß es unmuglich sei, ohne gute Werk selig werden.“20

So scharf drücken sich die Tübinger gegenüber dem Patriarchen nicht aus. Dieser hatte zu Art. 4-6 und entsprechend auch zu Art. 20 der Confessio Au-

19 Ep III, 4.

20 BSLK, 789, 17-21. 
gustana Graeca vermerkt, dass die reformatorische Lehre von der Rechtfertigung des Sünders aus Glauben nur dann ihre Richtigkeit habe, wenn unter diesem der lebendige, durch gute Werke bezeugte Glaube verstanden werde. Denn die Gnade werde dem nicht zuteil, der die nötige Bemühung missen lässt. Beide menschlicher Eifer und göttliche Begnadung - wirken miteinander und durchdringen sich gegenseitig. Zur Rechtfertigung vor Gott bedürfe es daher der vorhergehenden Buße ebenso wie der nachfolgenden Werke heiligender Liebe. Wer diese $\mathrm{zu}$ erbringen sich weigere, habe seinen himmlischen Lohn dahin. Zwar sollen wir unser Vertrauen keineswegs völlig auf unsere Gerechtigkeit setzen, da diese unvollkommen und der Gnade Gottes bedürftig sei, um ganz und integer zu werden. Gleichwohl müssen wir bestrebt sein, der uns durch Gottes Gnade zukommenden Hilfe entgegenzueilen und Fortschritte zu erzielen auf dem Wege der Heiligung. Ohne Werke tätiger Liebe kann von wahrem Glauben und wahrer Gerechtigkeit vor Gott nicht die Rede sein, auch wenn die Vergebung der Sünde uns anfänglich und bis auf Weiteres gratis und nicht unter Ansehung von Verdienst und Würdigkeit zugesprochen wird.

In ihrer Antwort vom 18. Juni 1577, die genau eine Woche vor Publikation der Konkordienformel am 50. Jahrestag der Übergabe des Augsburgischen Bekenntnisses erfolgte, pflichten die Tübinger Joachim II. bei, dass Buße sowie gute Werke zu tun zweifellos geboten sei und dass ein toter Glaube im Sinne bloßer Historienkenntnis nicht gerecht mache. Dies treffe nur für jenen Glauben zu, der durch die Liebe tätig sei. Dennoch erklären sie,

daß unsere guten Werke nicht mit dem Artikel von der Rechtfertigung durch Gott vermischt werden dürfen, wo es darum geht, wodurch und weshalb wir mit Gott versöhnt und zu Kindern und Erben Gottes gezählt werden. Warum? Weil wir dafür halten, dass je größerer Wert hier unseren Werken und Verdiensten beigelegt wird, desto geringer die Ehre der Tat Christi wird. ${ }^{21}$

Gerechtigkeit vor Gott und ewiges Heil verdanken wir allein der Gnade Gottes, wie sie in der Kraft des Heiligen Geistes in Jesus Christus, dem auferstandenen Gekreuzigten, offenbar ist. Die Rechtfertigung geschieht somit strictissime gratis, allein durch den Glauben und ohne alle Werke des Gesetzes, wobei unter Gesetz nicht nur das zeremoniale und zivile Gesetz, sondern die lex naturalis des Dekalogs zu verstehen ist. Weil aber die Früchte des Glaubens, die aus der in reiner Gnade empfangenen Rechtfertigung folgen sollen und tatsächlich folgen, unter irdischen Bedingungen stets unvollkommen sind und nie zur Vollkommenheit heranreifen, kann die Gerechtigkeit vor Gott nicht nur anfänglich, sondern auch

21 Acta et Scripta, 166. 
fernerhin nicht durch sie bedingt sein. Gewissheit des Heils kann der Glaube nur haben, wenn er sich ganz und gar und in all seinem Beginnen nicht auf gerechte Menschenwerke, sondern auf die Gerechtigkeit Christi verlässt. Deshalb hat es nach Tübinger Urteil seine Richtigkeit zu sagen, dass gute Werke weder der Bedingung- noch auch der Erhaltungsgrund der Gerechtigkeit sind, die vor Gott gilt.

Wenn demgegenüber der Patriarch im Mai 1579 zu bedenken gibt, dass von untätigem Glauben und glaubenslosen Werken zu reden gleichermaßen unsinnig und mit dem Christentum unvereinbar sei, so widersprechen die Tübinger dem zwar nicht einfachhin. Auch sind sie zu bekennen gerne bereit, dass diejenigen nicht Kinder Gottes heißen, die keine guten Werke tun. Ja selbst als empirisches Erkenntnismittel der Rechtfertigung sind sie die Werke des Glaubens unter gewissen Vorbehalten zu würdigen bereit. Gleichwohl bleibt es dabei, dass das Rechtfertigungsgeschehen als solches von Anfang bis Ende und in einer die Gesamtexistenz des Menschen umspannenden Weise allein durch Gnade und keinesfalls durch Menschenwerk bedingt wird. Wollen wir durch die Erfüllung des Gesetzes gerechtfertigt werden, wäre unser Heil dahin. Denn sind wir nach unserem natürlichen Vermögen als gefallene Menschen überhaupt nicht in der Lage, heilsame Werke zu tun, so können wir auch als Begnadete das Gesetz Gottes nur auf anfängliche und niemals vollkommene Weise erfüllen. Unser einziges Heil ist und bleibt daher Christus allein, der das Erlösungswerk nicht nur zum Teil, sondern ganz und gar vollbracht hat. Diese Vollgenügsamkeit der Gerechtigkeit Christi, auf die sich zu verlassen die Heilsgewissheit des Glaubens ausmacht, darf nicht durch falsches Vertrauen auf zwar gebotene, aber stets insuffiziente Werke des Gesetzes in Frage gestellt und in Zweifel gezogen werden. „Dem Mittler Christus allein und niemand anders gebührt die Ehre. Er selbst spricht durch den Propheten Jesaia: Ich, Ich tilge deine Übertretungen um Meinetwillen und gedenke deiner Sünden nicht. Niemand von uns ist jener Ich (Oudeis hemon esti [...] ho ego), der durch den Propheten spricht. Christus allein ist jener Mittler, der die Sünden abwäscht."'22 Das solus Christus und das sola gratia bilden sonach einen differenzierten Zusammenhang, worauf sich der Glaube gemäß dem Zeugnis der Schrift von der Rechtfertigung des Sünders verlässt. So bekennt es die Wittenberger Reformation in der Gemeinschaft des christlichen Glaubens.

22 Acta et Scripta, 309. 